\title{
Special Section: Oral Complications of Cancer Therapies
}

Guest Editor: Michael T. Brennan, DDS MHS 